\title{
Venlafaxina, un tratamiento eficaz para el trastorno de ansiedad generalizado
}

Gelenberg A; Lydiard R; Rudolph R y col.Efficacy of Venlafaxina Extended-Release Capsules in Nondepressed Outpatients with Generalized Anxiety Disorder. A six month randomized controlled trial. JAMA June 21, 2000. Vol 283. Nro 23.

\section{Objetivo}

Comparar la eficacia y seguridad de dosis variables de venlafaxina XR en pacientes ambulatorios con trastorno de ansiedad generalizado (TAG) sin trastorno del estado de ánimo.

\section{Diseño}

Ensayo clínico randomizado controlado con placebo a doble ciego en cuatro grupos paralelos con seis meses de seguimiento.

\section{Lugar}

Pacientes ambulatorios clínicos y de la practica psiquiátrica privada de 14 centros de los Estados Unidos.

\section{Pacientes}

Pacientes mayores de 18 años con TAG según los criterios de DSM IVR sin asociación con algún trastorno del ánimo o desorden severo de la personalidad y que estuvieran lo suficientemente sintomáticos como para recibir tratamiento.

\section{Intervención}

Los pacientes fueron randomizados y asignados a recibir placebo $(n=127)$ o tratamiento con venlafaxina $(n=124)$ a dosis de 50 , 150 y $225 \mathrm{mg}$ durante 28 semanas.

\section{Medición de los resultados principales}

Se evaluó a los pacientes con la escala de Hamilton y con la de impresión global de mejoría.

\section{Resultados principales}

La venlafaxina fue mejor que el placebo en forma estadísticamente significativa, lo que comenzó a notarse entre la primera $(p<0.02)$ y la segunda semana $(p<0.001)$. A la sexta semana la tasa de respuesta fue definitivamente mejor $(69 \%)$ en el grupo de venlafaxina que en el de placebo (42 a $46 \%$ ) con una $p<0.001$. A las 28 semanas, los puntajes de la escala de Hamilton fueron de -13.4 para los pacientes que habían recibido venlafaxina y de -8.7 para los del grupo placebo $(\mathrm{p}<0.001)$, mientras que la impresión global de mejoría fue de 2.2 mayor para venlafaxina XR $(p<0.001)$.

Los efectos adversos más frecuentes fueron la sequedad bucal, las nauseas y la somnolencia. Hasta los 56 días predominaron la constipación, los mareos, la sequedad bucal, las disfunciones sexuales ( $20 \%$ en varones y $8 \%$ en mujeres) las náuseas y la somnolencia. Entre los 56 y los 196 días muchos de ellos desaparecieron.

\section{Conclusiones}

Este fue el primer estudio que evaluó a más largo plazo el tratamiento del TAG con venlafaxina XR el cual mostró ser un fármaco seguro, de acción rápida y con la ventaja de poder ser usado en una sola toma diaria. El estudio demostró mejoría de los componentes psíquico y somático, proveyendo una alternativa para el tratamiento ansiolítico de corto y mediano plazo del TAG.

Fuente de financiamiento: Wyeth-Ayerst Research, Philadelphia. Pa.

\section{Comentario}

EI TAG tiene una prevalencia del $12 \%$ en los consultorios de atención primaria y una incidencia del 3\% anual en la población general. El que se exprese en forma mayoritariamente somática o cognitiva esta determinado culturalmente. La edad de inicio es aproximadamente a los 20 años y su curso, crónico y fluctuante. La venlafaxina XR es un antidepresivo dual que actúa sobre la inhibición de la recaptación de serotonina (IRS) y de noradrenalina en forma simultánea (este tipo de acción sobre mas de un sistema aminérgico busca mayor eficacia). Presenta una curva dosisrespuesta sigmoidea, aumentando la magnitud de su efecto desde los 75 a los $225 \mathrm{mg} /$ día y tendiendo luego a estabilizarse o decrecer. Se comporta como un IRS a dosis bajas (hasta 150mg/día) presentando características duales a altas dosis (máxima de $225 \mathrm{mg} / \mathrm{dí}$ ). Los efectos dependientes de la noradrenalina (sudoración, temblor, hipertensión arterial diastólica) aparecen ạ altas dosis, presentándose la HTA dentro de los dos meses de estabilización de la dosis.

El reciente interés por encontrar tratamientos alternativos a las benzodiacepinas para el TAG nos lleva a comparar la eficacia entre éstas y los antidepresivos, y placebo. En este estudio el $61 \%$ de los pacientes recibieron entre 100 y $200 \mathrm{mg} /$ día de venlafaxina XR y un $10 \%$, mas de $200 \mathrm{mg} /$ día. El $59 \%$ de los pacientes (147/251) quedó fuera de tratamiento durante la fase dual, básicamente por una respuesta insatisfactoria, lo que produjo una pérdida significativa de pacientes en el grupo de venlafaxina. Los efectos adversos fueron responsables del $26 \%$ de las suspensiones (el incremento de la tensión arterial fue el motivo en el $4 \%$ ).
Según nuestra experiencia los cambios reportados en las escalas de Hamilton e índice global de mejoría son clínicamente significativos y se relacionan con mejoría subejtiva en los pacientes.

Un estudio controlado randomizado y doble ciego de ocho semanas de duración comparó trazodone a una dosis máxima de $255 \mathrm{mg} /$ día con placebo, diazepán a una dosis máxima de $25 \mathrm{mg} /$ día e imipramina a dosis máxima de $143 \mathrm{mg}$ día. Este ensayo sugirió la importancia del rol de los antidepresivos, en especial la imipramina, para el tratamiento del TAG y encontró diferencias significativas entre la eficacia de los tres grupos con relación al placebo 1 .

Otro estudio compara la eficacia entre diazepám, paroxetina e imipramina y halló que a partir de la cuarta semanas ambos antidepresivos mostraban mejorías mayores que el diazepám, que los antidepresivos mejoraban el cuadro, mayoritariamente a expensas del componente psíquico, mientras que la benzodiazepina lo hacía en función del componente somático. El estudio sugirió que paroxetina es efectivo en el tratamiento del TAG2

Teniendo en cuenta que a dosis bajas ( 75 a $150 \mathrm{mg} / \mathrm{dia}$ ) la venlafaxina se comporta básicamente como un IRSS, sería importante evaluar en forma comparativa la eficacia de un IRSS con venlafaxina XR para verificar si la acción dual de esta última, le otorga mayor eficacia. Sería interesante, además, realizar estudios de seguimiento mas prolongado para evaluar la eficacia comparada entre placebo, diazepam, imipramina y venlafaxina en cuanto a la prevención de episodios futuros, ya que se trata de una patología crónica y recurrente.

Dra. Mónica Viviana Santagata [ Especialista Universitaria en Psiquiatría Coordinadora del Programa de Asistencia Medica Transcultural Hospital Italiano de Buenos Aires ]

\footnotetext{
Referencias

Rickels K; Downing R; Schweizer E; Hassman H. University of Pennsylvania of Philadelphia. Antidepressants for the tratment of generalized anxiety. A placebo controlled comparison of 1. Rickels K; Downing R; Schweizer E; Hassman H. University of Pennsylvania of Philad
imipramine, trazodone and diazepam. Arch Gen Psychiatry 50 (11) $884-951993$ Nov. 2. Rocca P; Fonzo V; Scotta M; Zanalda E; Ravizza L. Paroxetine efficacy in the treatment of generalized anxiety disorder. Department of Neoroscence University of Turín, Italy. Acta Psychiatr. 95(5): 444-50 1997 May.
} 